\title{
VIOLENCIA DE GÉNERO. FORMACIÓN E INTERVENCIÓN DESDE LA INGENIERÍA
}

\section{GENDER VIOLENCE. TRAINING AND PREVENTION FROM AN ENGINEERING POINT OF VIEW}

\author{
Josefa Andrea Leva Ramírez*, Mª Joaquina Berral Yerón \\ $\mathrm{M}^{a}$ Victoria García Gómez, Inmaculada Serrano Gómez
}

*me1leraj@uco.es

\begin{abstract}
Resumen
La violencia de género sí tiene género. Es un fenómeno que suscita la mayor reprobación social y que está muy lejos de ser erradicado. Constituye uno de los vértices de la violencia machista y por tanto mientras la igualdad de género no sea una realidad continuará existiendo. Este artículo describe el proyecto llevado a cabo por un grupo de docentes y de estudiantes de ingenierías de la Escuela Politécnica Superior de Córdoba que, a través de talleres, practicaron competencias de comunicación y adquirieron a la vez conocimientos sobre herramientas de lucha contra la violencia de género, tanto desde el ámbito personal como laboral.
\end{abstract}

Palabras clave: Violencia de género; Ingeniería; Competencias.

\section{Abstract}

Gender violence does have gender. It is a phenomenon that provokes on one hand great social outcry, but on the other is still far from being eradicated. It is one of the cornerstones of sexist violence and a consequence will continue to exist if gender equality is not a reality. In this work, a group of teachers and students of the School of Engineering Sciences have collaborated through workshops to improve the situation. By practicing communication skills, these students gained knowledge about tools to combat gender violence, both personally and within a work/team environment.

Keywords: Gender violence; Engineering; Skills.

\section{INTRODUCCIÓN}

Cada vez que una mujer es asesinada por su pareja o ex pareja estalla un aldabonazo en nuestras conciencias, sin embargo la violencia de género se ha convertido en un fenómeno cotidiano (GIL). La discriminación y desigualdad de trato que sufren las mujeres es la causa de la violencia de género, (VG en lo sucesivo), siendo la expresión más cruenta de esta situación. Será preciso aunar esfuerzos y trabajar en todos los entornos para poco a poco ir cambiando esta realidad. ¿Podemos hacer algo desde nuestras aulas? ¿Es nuestra responsabilidad?

En nuestro ámbito profesional somos docentes de distintos Grados de Ingenierías. Nuestros estudiantes son personas con una gran capacidad de trabajo y dedicación, muy centrados en su preparación para su futuro laboral y alejados de movimientos sociales, políticos o reivindicativos en general.

No es que no les preocupen los asesinatos, es que piensan, al igual que parte de los responsables de los estudios universitarios, que la formación en género, precisa para acabar con las violencias machistas y avanzar en igualdad, es algo que debe estar recogido en los planes de estudios de los títulos "que proceda". ¿Cuáles son en los que procede? ¿En estudios de ingeniería procede? ¿Profesionalmente la sociedad les exigirá a nuestros ingenieros e ingenieras esos conocimientos?

Sí. Sin ninguna duda.

Nuestros estudiantes en numerosas ocasiones forman parte de los equipos directivos de las industrias y empresas, si bien mayoritariamente desempeñarán puestos intermedios de mando, estando al frente de equipos de trabajo generalmente muy masculinizados. En este contexto, será preciso poseer los conocimientos suficientes para poder gestionar las relaciones y entender y atender las necesidades de un grupo tan diverso como la sociedad actual. De sus conocimientos dependerá en gran medida el buen funcionamiento del equipo.

Por otra parte, sus actitudes, comportamientos o comentarios encontrarán un amplificador en cada uno de los componentes, ayudando a generar dinámicas positivas hacia la igualdad y el respeto.

En cuanto a legislación que nos sitúe, la L.O. 1/2004, de 28 de diciembre, de Medidas de Protección Integral contra la Violencia de Género, conocida como "Ley de protección integral sobre la Violencia de Género" representó para el común de los españoles la exigencia para que la sociedad asumiera su responsabilidad en la lucha contra la VG y la protección de sus víctimas.

Esta ley, junto a la L.O. 3/2007, de 22 de marzo, para la Igualdad Efectiva de Mujeres y Hombres, conocida asimismo como "Ley de igualdad", constituyó el gran cuerpo normativo conocido generalmente por las personas ajenas al campo jurídico. 
Es a partir de la aplicación de esta última ley cuando el campo de la empresa en general, y de la ingeniería en particular, comenzó a ser consciente de la necesidad de aplicar medidas reales en las empresas que favorecieran la progresiva igualdad entre géneros, comenzando a elaborarse los primeros planes de igualdad (inicialmente solo en empresas de más de 250 trabajadores). Es también en esta ley donde aparecieron de manera explícita las primeras recomendaciones a las empresas, de realización voluntaria, de acciones de Responsabilidad Social (RS en lo sucesivo) en materia de igualdad.

La RS Empresarial o Corporativa, aunque con retraso, está calando hondo en la sociedad española (NAVARRO), las empresas cada vez son más conscientes de que es un valor añadido, con repercusión directa en sus cuentas de resultados a través del buen nombre de sus marcas. La publicación en julio de 2014 de la "Estrategia Española 2014 - 2020 sobre Responsabilidad Social de las Empresas" y su importante campaña de comunicación representó la mayor apuesta institucional del gobierno español por esta herramienta.

Todo lo anterior, a lo que se podría añadir, entre otros, conceptos contemplados en la Ley 31/1995, de 8 de noviembre, de Prevención de Riesgos Laborales, nos condujo a un escenario en el que se puede asegurar, desde nuestro punto de vista, que la lucha contra la VG y por la igualdad, así como la formación en género es procedente y necesaria en cualquier Plan de Estudios de Ingeniería.

Llegados a ese punto resultó lógico plantearse cómo abordar esta formación dentro de nuestras asignaturas. La solución decidida fue cubrir a la vez otra necesidad, también perentoria: reforzar la formación en competencias de comunicación recogidas en los correspondientes títulos. Estas competencias básicamente consisten en expresar problemas, argumentar posibles soluciones y discutir ideas, tanto ante un público especializado como no especializado. Reforzando la adquisición de estas competencias utilizando como materia de aprendizaje la relacionada con la VG, RS o género, conseguimos hacer un planteamiento que capte a nuestro estudiantado y lo sume como agente activo hacia la igualdad.

\section{OBjeTIVOS}

Los objetivos prioritarios de este artículo son los referentes a la temática del proyecto que describe, si bien hay que reconocer la importante aportación al mismo del trabajo en competencias básicas. Concretamente hemos perseguido:

- Sensibilizar al estudiantado acerca de la gravedad y extensión de la Violencia de Género.

- Iniciarlos e iniciarlas en el estudio de la igualdad de género como elemento generador de libertad, también para varones.

- Dar visibilidad al tejido social cordobés que lucha contra la VG.

- Reconocer la RS empresarial, sus memorias y los documentos de conducta o éticos como herramientas útiles para luchar contra la VG y por la igualdad desde el ámbito empresarial e industrial.

- Reforzar la adquisición de las competencias básicas relacionadas directamente con la comunicación, contempladas entre otras en las asignaturas implicadas en la experiencia. En nuestros planes de estudio son las denominadas $\mathrm{CB} 2^{1}$ y CB $4^{2}$.

\section{Metodología}

El proyecto descrito en este artículo se llevó a cabo con las docentes y estudiantes de tres asignaturas de tres grados distintos: Grado en Ingeniería Mecánica (GIM), Grado en Ingeniería en Electrónica Industrial (GIEI) y Grado en Ingeniería Informática (GII), y con la colaboración adicional de tres asignaturas del GIM y dos asignaturas del GIEI.

De acuerdo a los objetivos perseguidos y al tipo de competencias a trabajar, unas instrumentales tales como las competencias de comunicación y otras actitudinales, como el compromiso ético, apreciación de la diversidad y trabajo en equipo, se acordó abordar esta docencia desde metodologías activas fuertemente participativas.

Se decidió seguir de manera general la siguiente estructuración básica:

- Con una semana de antelación a la realización del taller facilitar a través de Moodle (la platarforma virtual de la UCO) material de consulta y trabajo sobre el tema concreto a tratar, para que pudiese ser consultado previamente por los estudiantes y las estudiantes.

- Comenzar el taller con una presentación de la persona experta invitada, de unos 35 minutos, para posteriormente abrir un turno de preguntas.

- A continuación, trabajar por grupos a propuesta del ponente.

- Posteriormente discutir y debatir opiniones colectivamente.

${ }^{1}$ CB2: Que los estudiantes sepan aplicar sus conocimientos a su trabajo o vocación de una forma profesional y posean las competencias que suelen demostrarse por medio de la elaboración y defensa de argumentos y la resolución de problemas en el campo de la Ingeniería.

${ }^{2}$ CB4: Que los estudiantes puedan transmitir información, ideas, problemas y soluciones a un público tanto especializado como no especializado. 
- Para concluir el taller, por grupos, elaborar durante 5 minutos una reflexión final y exponerla con brevedad a modo de cierre en las conclusiones finales de la sesión.

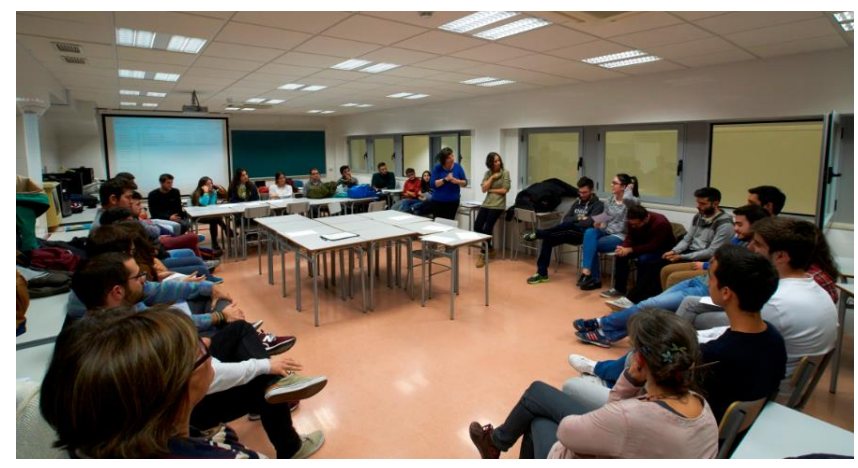

1. Momentos de puesta en común final de un taller.

El desarrollo del proyecto comenzó el 21/09/2016 con una reunión del grupo docente y colaboradores, dedicada a la organización de aspectos puntuales del proyecto y apertura en Moodle de un espacio propio para el proyecto.

A lo largo de la semana siguiente se presentó la propuesta dentro de las asignaturas "Mecánica de Materiales" en GIM y GIEI. Al estudiantado de GII se les presentó el proyecto a partir del segundo cuatrimestre. Se facilitó a todas las personas presentes ese día en las correspondientes aulas una encuesta similar a la del CIS no 2992 sobre "Percepción Social de la VG por la Adolescencia y la Juventud" y se indicó que si no les resultaba violento, la devolviesen contestada en los días sucesivos. El resto de hitos más relavantes fueron los siguientes:

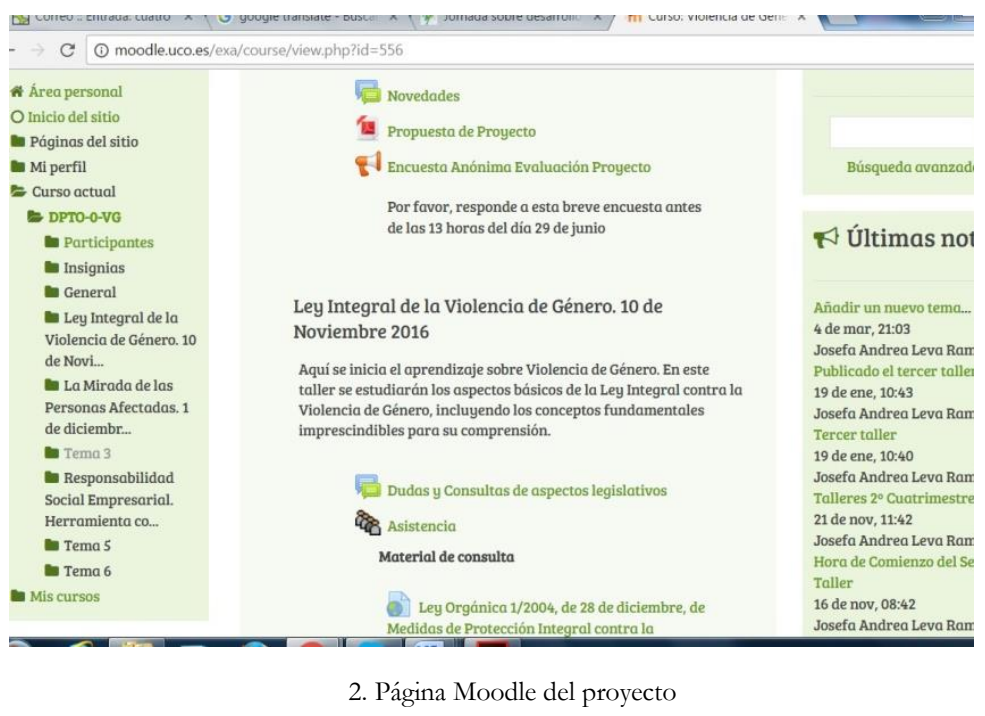

(05/10/2016) Segunda reunión en la que el equipo docente analizó el pequeño número de encuestas respondidas, prácticamente coincidentes con el número de estudiantes implicados inicialmente en el proyecto.

(02/10/2016) Se subió a la plataforma la documentación sobre la que se trabajó el 10/10/2016 en el taller liderado por D. Luís Manuel Rodríguez Osuna. Bajo el título "Desmontando mitos sobre la Ley Integral contra la Violencia de Género" se realizó una aproximación a la legislación existente al respecto. 


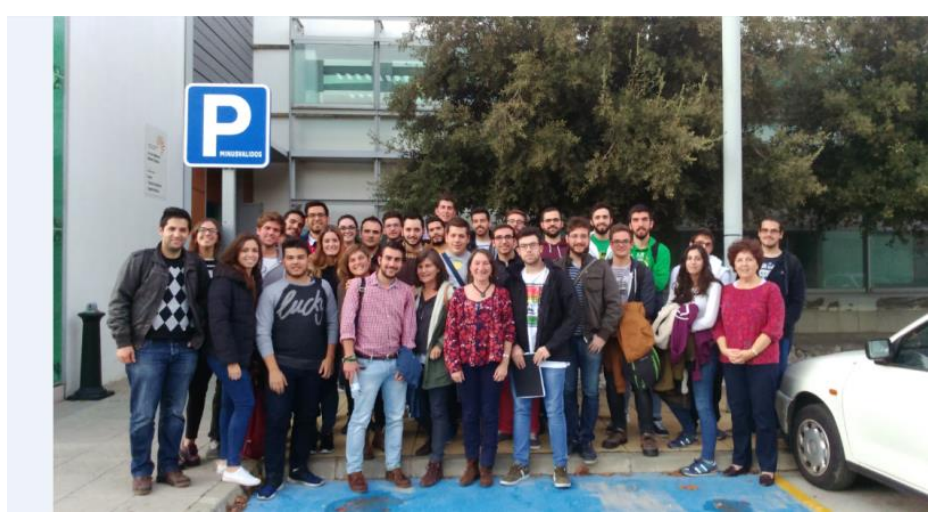

3. Foto de familia del primer taller.

(24/11/2017) Se subió el material para el taller "La mirada de las afectadas por la Violencia de Género", celebrado el 01/12/2016 a cargo de Dña. Antonia Mudarra Lara de Feminismos Córdoba, y Dña. Elena Vega Horcas de la Plataforma Cordobesa contra la Violencia hacia las Mujeres. Este taller resultó especialmente enriquecedor dado que produjo en los participantes un cambio de percepción respecto a la extensión y virulencia de la Violencia de Género en nuestro entorno cercano. Se llegó a la comprensión de que la violencia de género es la expresión más cruel y humillante de un orden cultural que se basa en la violencia no solo física, sino también simbólica (SALAZAR), fue por ello sumamente importante abordar el estudio de la terminología específica. Se trató con mucha extensión, como no podía ser de otro modo, los sentimientos de las distintas personas afectadas: mujeres víctimas de VG, hijos, padres, madres, familiares, amigos...

(23/02/2017) Se celebró el taller “Detección de Violencia de Género y abordaje de la situación”, en el que se contó con la participación de Dña. Carmen Salido Fernández, psicóloga del centro en Córdoba del Instituto Andaluz de la Mujer, y con la preparación previa del equipo docente a cargo de la psicóloga participante en el proyecto Dña. Laura Moya Berral. Dentro de esta sesión se dieron a conocer las herramientas con las que cuenta el Instituo Andaluz de la Mujer para dar apoyo a las mujeres adolescentes, mujeres adultas e hijos e hijas. Fue especialmente relevante dentro de la sesión descubrir que la VG tiene su aparición en muchos casos en edades realmente tempranas, siendo imprescindible abordar la situación con las herramientas adecuadas.

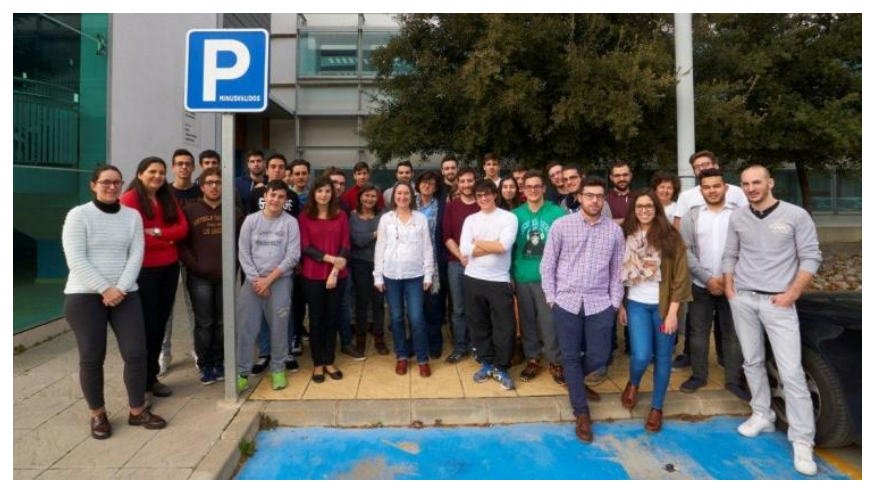

4. Foto de familia del tercer taller.

(30/03/2017) Tuvo lugar el último taller. Dedicado al estudio de la RS corporativa como herramienta contra la VG, liderado por Dña. Elena Quintas Robles, directora de RRHH de la zona sur del Grupo Votorantim Cimentos, con el apoyo de Dña. Cecilia Gómez Morgollón, responsable de comunicaciones en la zona sur del mismo grupo. La preparación previa del grupo docente para esta sesión corrió a cargo de la ingeniera Dña. M Luz Guillén y el ingeniero D. Juan Báez, a pesar de que por motivos laborales no pudieron estar en el desarrollo de la actividad.

(10/06/2017) Se cursó invitación a participar en el taller "Nuevas Masculinidades", organizado por la asociación Mujeres en Zona de Conflicto en su cede en Córdoba, y liderado por D. Eric Pescador. A pesar de la evidente calidad de la oferta, el estudiantado en ese momento estaba en plenos exámenes, por lo que la asistencia del grupo fue exigua. 


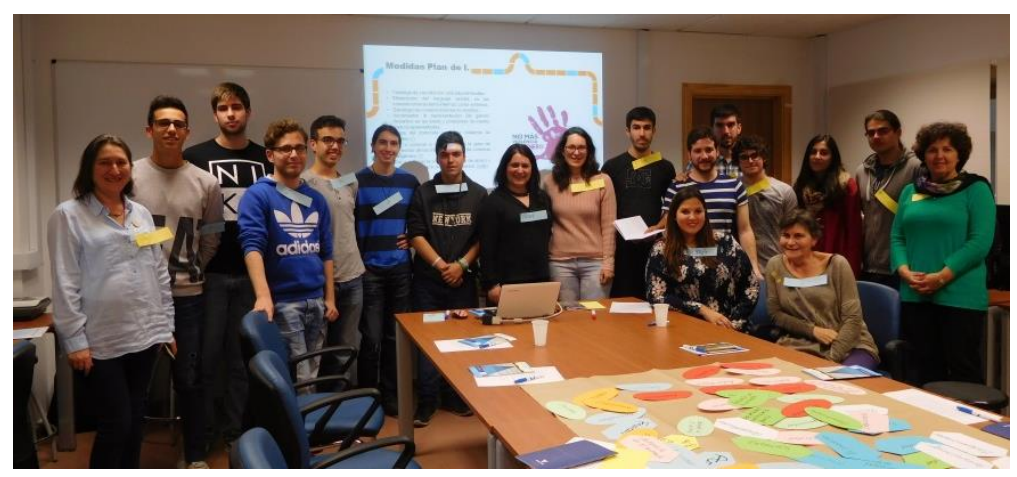

5. Momentos finales del cuarto taller

(16/06/2017) Reunión final del grupo docente y valoración global del desarrollo del proyecto.

\section{RESULTADOS OBTENIDOS}

El alumnado pudo realizar una encuesta anónima, vía Moodle, sobre el proyecto entre los días 26 y 29 de junio, donde se reflejó que mayoritariamente estaban muy satisfechos y satisfechas con los talleres. Los resultados más representativos son los siguientes:

- Todos los estudiantes piensan que no debe eliminarse ni modificarse ningún taller de los celebrados.

- Sobre la formación recibida, el $80 \%$ la valora con $4 / 5$ y el $20 \%$ con $5 / 5$.

- En cuanto a transferencia al entorno profesional, el $20 \%$ la valora con $3 / 5$, el $53 \%$ con $4 / 5$ y el $27 \%$ con $5 / 5$.

- En cuanto a transferencia a la vida privada, el $6,7 \%$ la valora con $3 / 5$, el $26,7 \%$ con $4 / 5$ y el $66,6 \%$ con $5 / 5$.

- Sobre el interés en ofertar el proyecto a nuevos estudiantes el próximo curso, el $13,3 \%$ lo valora con 3/5, el $6,7 \%$ con $4 / 5$ y el $80 \%$ con $5 / 5$.

- A la pregunta de si quieren ampliar su formación con un nuevo proyecto el próximo curso, el $6,7 \%$ lo evalúa con $2 / 5$, el $20 \%$ con $3 / 5$, el $13,3 \%$ con $4 / 5$ y el $60 \%$ con $5 / 5$.

- A la pregunta de si a lo largo de este tiempo han podido poner en práctica algo de lo aprendido en los talleres, un $60 \%$ ha contestado afirmativamente.

Desde otro punto de vista, la opinión de las personas colaboradoras en los talleres fue sumamente positiva, nos animaron a seguir es esta línea y se comprometieron a participar en futuras actividades.

La valoración del grupo docente también fue muy positiva. El haber aportado este tipo de formación a nuestros y nuestras estudiantes supondrá un valor añadido para su futuro. Cómo dice nuestra compañera Dña. Amelia Sanchís Vidal, "incluir la transversal de género y feminismo puede suponer un antes y un después en la formación."

\section{UTILIDAD/ANÁLISIS}

Consideramos sumamente importante que los futuros ingenieros e ingenieras posean este tipo de formación que les capacite para ser agentes activos de cambio tanto desde sus facetas privadas como públicas, colaborando activamente a hacer una sociedad más incluyente, corresponsable e igualitaria. Pensamos que sería deseable que en otras escuelas de ingeniería se trabajasen también aspectos de igualdad y perspectiva de género. 


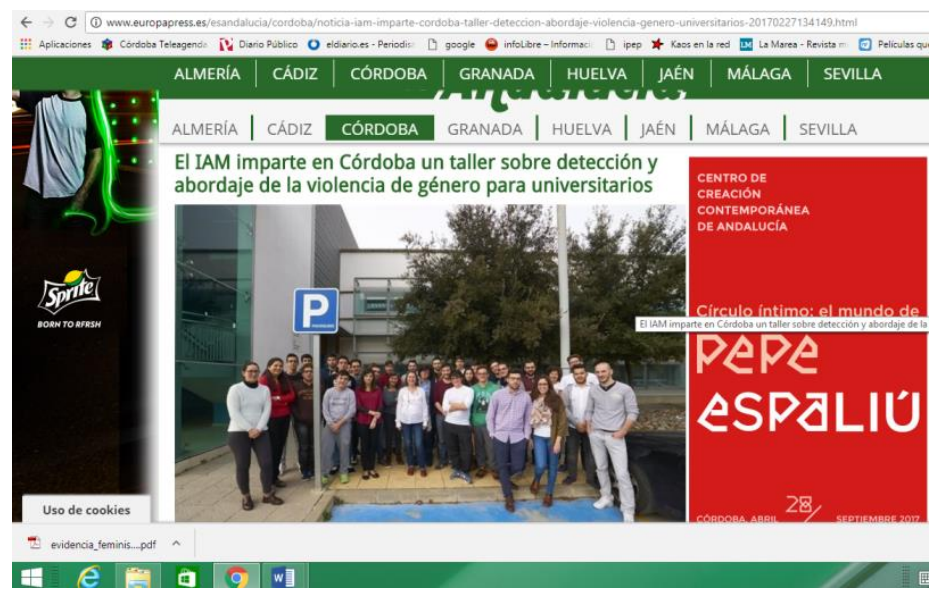

\section{CONCLUSIONES/DISCUSIÓN}

Hemos comprobado que docentes y discentes podemos aprender juntos y juntas sobre estos temas, reconociéndonos como miembros de una comunidad preocupada y ocupada en aspectos sociales de actuación, que por otra parte hacen a la empresa en la que trabajamos mejor.

Proyectos de este tipo deberían de estar incluidos en la formación reglada de los estudiantes universitarios. El tema es suficientemente importante como para no dejar que su aprendizaje se realice de forma voluntaria.

\section{BIBLIOGRAFÍA}

1. Gil Rodríguez, E. LLoret Ayter, I. Pujal i LLombart, M. Violencia de género y feminismo Editorial UOC ISBN:9788497886277

2. Navarro García, F. Responsabilidad Social Corporativa: Teoría y Práctica. Esic Editorial. ISBN: 9788473565288

3. Salazar Benítez, O. Masculinidades y Ciudadania. S.L. - DYKINSON. ISBN: 9788490313961

4. De Miguel Luken, V., Percepción de la Violencia de Género en la Adolescencia y la Jwventud, Ministerio de Sanidad, Servicios Sociales e Igualdad. ISBN: 978-84-7670-728-9 [Online]. Available:

http://www.violenciagenero.msssi.gob.es/violenciaEnCifras/estudios/investigaciones/2015/pdf/Libro20 Percepc ion Social VG .pdf 